\title{
The influence of uterine artery embolisation on ovarian reserve, fertility, and pregnancy outcomes $-\mathrm{a}$ review of literature
}

\author{
Piotr Czuczwar, Anna Stępniak, Wojciech Wrona, Sławomir Woźniak, Paweł Milart, Tomasz Paszkowski \\ $3^{\text {rd }}$ Department of Gynaecology, Medical University of Lublin, Lublin, Poland
}

\begin{abstract}
Uterine fibroids are considered to be the most frequent female benign tumours. Fibroids affect mainly women of reproductive age. The most frequently reported signs and symptoms of fibroids include disturbances of the menstrual cycle such as heavy bleeding and painful menstruation, pelvic masses associated with pelvic pain, urinary problems or constipation, as well as infertility and recurrent pregnancy loss. The mainstay of fibroid treatment is still surgery. However, many patients seek alternative treatment options for fibroids, to preserve their uterus and fertility. One of the most important alternative treatment options for fibroids is uterine artery embolisation (UAE). However, there are some concerns that UAE may negatively influence ovarian function and even result in premature menopause. Moreover, the use of UAE in patients with future reproductive plans is still controversial, due to the possible pregnancy complications. The purpose of this review is to summarise the current knowledge regarding the possible influence of UAE on fertility, pregnancy outcome, and ovarian reserve.
\end{abstract}

Key words: uterine artery embolisation, fibroids, ovarian reserve, fertility.

\section{Introduction}

Uterine fibroids affect about $50 \%$ of women in reproductive age. Fibroids, also known as myomas or leiomyomas, are benign tumours originating from smooth muscles tissue, and they are considered to be the most frequent female benign tumours. Their occurrence increases with age and is highest around menopausal age, after which it decreases continuously. The symptoms of fibroids vary depending on the size and location of the tumour; however, the vast majority of the tumours are asymptomatic and are incidental findings during routine gynaecological examination (bimanual or ultrasound), which do not require any treatment beyond observation. The most frequently reported signs and symptoms of fibroids include disturbances of the menstrual cycle such as heavy bleeding and painful menstruation, pelvic masses associated with pelvic pain, urinary problems, or constipation. Infertility and recurrent pregnancy loss may also be caused by uterine fibroids, especially submucous and intramural [1]. Donnez in his recent article mentioned several mechanisms of fertility impairment in females with fibroids: changes in the normal uterus anatomy, alterations in uterus function (increased contractility, flawed blood supply), and local hormone changes induced by fibroids [2]. Obstetric complications might appear as preterm delivery, abnormal presentation, lower infant birthweight, as well as incidence of short cervix [3]. If ailments develop, uterine fibroids will require precise assessment and individual treatment plan proposal. There is a wide range of therapeutic options, including surgical treatment, non-surgical alternatives, and medical therapy, for the management of fibroids. Originally, hysterectomy performed by laparotomy was considered as a treatment of choice in symptomatic fibroids. Nowadays the approach to uterine fibroma treatment has been progressively changing due to increased concern about patient's quality of life and individualised treatment expectations. Current management is based mostly on surgical intervention; however, because other treatment options are available, the choice of the therapy is guided by the patients' age, and their wish to avoid surgery and to preserve the uterus. According to a national survey performed by Borah et al. almost $30 \%$ of 968 women included in the study reported missing work and limitation of their career potential due to the symptoms of fibroids [4]. Fibroids may cause depression and severe deterioration of quality of life among females. Fear of uterus loss is one of the main reasons why women postpone treatment for about four years after recognition. In the survey almost 50\% of the respondents expressed a desire to preserve the uterus and fertility [4]. Most women preferred non-invasive options for treatment regardless of their desire for childbearing. Interestingly, uterus-sparing treatment 
options were important to women regardless of their future reproductive plans [4].

In spite of surgical intervention, less-invasive management methods of uterine fibroids are available. Pharmacological treatment, with effectiveness resulting from the oestrogen and progesterone dependency of the tumours, includes $\mathrm{GnRH}$-agonists, progesterone receptor antagonist - mifepristone, and selective progesterone receptor modulators - ulipristal acetate. Minimally invasive techniques have become another option for symptomatic fibroid treatment, including uterine artery embolisation (UAE), transvaginal uterine artery occlusion, magnetic resonance imaging-guided focused ultrasound, percutaneous laser ablation, and cryoablation. Although most of the methods mentioned above are rather costly and are not performed on a wide scale, UAE is definitely the most commonly used procedure, and since its introduction in 1995 it has become the treatment of choice in women wishing to preserve the uterus [5]. During UAE embolic material occludes both uterine arteries, which is achieved by introducing the catheter through the femoral artery into uterine arteries under X-ray guidance. As a result, blood flow is significantly reduced and ischaemia appears in the tumour, causing necrosis of fibroid cells. Comparing UAE to surgical intervention, the hospital stay and recovery period are shorter, complications occur less frequently and are mostly associated with post-embolisation syndrome [6]. As the main group of patients qualified for UAE consists of females over 40 years of age or women without further maternity plans, most studies recognised this method as potentially safe and focused mostly on its benefits, while recently more researchers focused on assessing the impact of UAE on fertility. At present, professional societies like the American College of Obstetrics and Gynaecology (ACOG) and the Society of Interventional Radiology (SIR) list the desire for future fertility as a relative contraindication to UAE [7]. This article will focus on the influence of UAE on determinants of fertility, such as ovarian function and pregnancy outcomes after embolisation, in women of reproductive age.

\section{Uterine artery embolisation and pregnancy}

Theoretically, UAE may affect fertility by many different mechanisms. Ideally, reducing blood flow in the uterine arteries should lead to irreversible ischaemic injury of fibroid cells and necrosis in the tumour, while normal myometrium should be able to recover (Aitken et al. 2006; Banu et al. 2007). However, ischaemic injury within the myometrium cannot be excluded. After UAE fibroids undergo necrosis and they shrink, but they do not disappear. The presence of necrotic tissue within the uterine wall may increase the risk of uterine rupture during pregnancy. The remaining fibroid mass may be responsible for pregnancy complications related to the presence of fibroids. Finally, it has been suggested that UAE may impair ovarian function and thus either decrease the chances of achieving pregnancy or result in premature menopause [8]. Unfortunately, investigating the influence of UAE on fertility and pregnancy outcome may be difficult. Because the incidence of fibroids is greatest during the menopausal transition, most patients with fibroids have completed their reproductive plans. This results in relatively small numbers of patients who still wish to conceive after fibroid treatment and could be included in studies investigating these issues. Additionally, due to the fact that after UAE the fibroid mass remains present in the uterus it may be difficult to distinguish whether the observed complications are the result of the procedure itself or the presence of remaining fibroid tissue.

The impact of UAE on conception and obstetric complications remains controversial. Various studies were performed to assess whether UAE should be recommended for women in childbearing age with extensive fibroids or it should be dedicated only to patients without further maternity plans. Complications of pregnancy after UAE, such as increased rate of miscarriage, preterm delivery, intra-uterine growth restriction, malpresentation, abnormal placentation, and post-partum haemorrhage were enlisted in the study by Holub et al. [9]. According to Goldberg and Pereira, pregnancies following uterine artery embolisation had higher rates of preterm delivery (OR 6.2, 95\% Cl: 1.4-27.7) and malpresentation (OR 4.3, 95\% Cl: 1.0-20.5) than pregnancies following laparoscopic myomectomy [10]. Conversely, Homer and Saridogan found similar rates of preterm delivery $(14 \%$ vs. $16 \%$; OR 0.9; $95 \% \mathrm{Cl}$ : $0.5-1.5)$, IUGR (7.3\% vs. $11.7 \%$; OR $0.6 ; 95 \% \mathrm{Cl}: 0.3-1.3)$, and malpresentation (10.4\% vs. $13 \%$; OR $0.8 ; 95 \% \mathrm{Cl}: 0.4-1.5)$ in UAE pregnancies and in control pregnancies with fibroids [11]. However, the authors confirmed the increased risk of miscarriage, caesarean section, and post-partum haemorrhage in pregnancies after UAE [11].

Some authors strongly support the idea of performing UAE in patients seeking future fertility. McLucas et al. claim that the abovementioned pregnancy complications are associated with fibroids in general, and since UAE does not diminish fibroids totally, fertility outcomes may be less favourable than those after myomectomy [12]. These authors also showed high pregnancy rates after UAE (up to 48\%) [13]. Mohan et al. [14] analysed 21 studies that reported pregnancy outcomes or complications of pregnancy following UAE for fibroid tumours. The cumulative pregnancy rate and miscarriage rate following UAE for fibroid tumours from the pooled analysis were $58.6 \%$ and $28 \%$, respectively. The mean age of women was 35.9 years. The cumulative live birth rate was $65.2 \%$, with a term delivery rate of $60.7 \%$. The mean pre-term delivery and placental abnormality rates 
were $7.3 \%$ and $6.3 \%$, respectively [14]. The authors suggested that pregnancy rates following UAE are comparable to age-adjusted rates in the general population [14]. Such findings definitely prove that pregnancy after UAE is possible and is not uncommon. However, it should be stressed that in patients with fibroids fertility problems are caused mainly by the presence of the tumours and not only by the intervention itself. Comparing the results obtained in different populations does not allow us to draw meaningful conclusions because the initial fibroid volume and location could have been different and might have influenced the final reproductive outcome. Therefore, randomised studies directly comparing various treatment options for fibroids are necessary, to be able to choose the best treatment option when considering future fertility. Currently, only one randomised study comparing fertility outcomes of UAE with laparoscopic myomectomy is available [15]. Twenty-six patients after UAE and 40 after myomectomy tried to conceive, with a pregnancy rate of $50 \%$ vs. $78 \%(p<0.05)$ and miscarriage rate of $53 \%$ vs. $19 \%$ ( $p<0.05)$ for UAE and myomectomy, respectively. The authors concluded that myomectomy had superior reproductive outcomes in comparison to UAE [15]. Unfortunately, the study had some limitations, such as small sample size, short duration of follow-up, and high rate of re-intervention in the UAE group. Finally, a recent Cochrane Database review addressed the issue of UAE and fertility. The review revealed that there is some indication that UAE may be associated with less favourable fertility outcomes than myomectomy (live birth: OR 0.26; 95\% Cl: 0.08-0.84; pregnancy: OR 0.29; 95\% $\mathrm{Cl}$ : 0.10-0.85), but these data should be regarded with extreme caution [16].

Another issue raised by the enthusiasts of performing UAE in patients planning future pregnancies is the risk of fibroid recurrence after myomectomy. It has been found that in younger patients after myomectomy the risk of repeat surgery due to fibroid recurrence may be as high as 44\% [17]. That is why it is advised to conceive within six months after myomectomy, because during this time fibroids are less likely to recur. It has been suggested that after UAE patients do not have to limit their reproductive plans to the six-month time window after the procedure, which may be beneficial for younger patients who do not wish to conceive immediately after treatment [12]. On the other hand, long-term observations show that after UAE over a quarter of patients will also require further surgical interventions [18].

UAE has also been offered to patients wishing to conceive, who did not qualify for a myomectomy. In 2014 Torre et al. conducted a very interesting study, in which 31 women who had extensive symptomatic fibroids and were not eligible for surgery were prospectively followed-up after UAE and were actively trying to conceive. In the whole group only one pregnancy occurred, and it ended in a miscarriage. The authors concluded that UAE should not be performed in young women of childbearing age with extensive fibroids [19]. Additionally, the authors reviewed the literature from 1998 to 2013, identifying 738 women expressly wishing to conceive at the time of UAE. Fertility, miscarriage, and take-home baby rates of $36.3,29.7$, and $19.6 \%$, respectively, were calculated [19]. The results of the study mentioned above are worse than those already reported in women wishing to conceive after UAE.

\section{Uterine artery embolisation and ovarian reserve}

Ovarian reserve (OR) is a measure of individual reproductive potential, understood as the number and quality of remaining oocytes. OR is used mainly to predict the response to ovarian stimulation. Decreased $\mathrm{OR}$ is sometimes referred to as primary ovarian insufficiency and has to be distinguished from menopause and premature ovarian failure [20]. It is believed that decreased OR results in poor ovarian response (decreased number of follicles, decreased number of embryos, and increased cancelation rates) as well as in reduced fecundability. However, it should be stressed that currently there is no uniformly accepted definition of decreased OR [21]. Biochemical and ultrasound tests allow an indirect assessment of OR. The biochemical tests include follicle-stimulating hormone (FSH), estradiol, inhibin $\mathrm{B}$, and antim llerian hormone $(\mathrm{AMH})$ and the clomiphene citrate challenge test. The antral follicle count (AFC) and ovarian volume are the ultrasound tests that are used to assess OR. According to current guidelines, FSH is the most commonly used screening test for decreased OR, but AFC and AMH are the most promising predictors [21].

Many factors, such as chemo- or radiotherapy and tobacco smoking may have a negative impact on OR [22]. It has also been hypothesised that during UAE the embolic material may reach the ovarian circulation and diminish ovarian function [23]. And indeed, embolic material was found in the blood vessels supplying the ovaries in patients who underwent UAE [24]. The theoretical rationale for this phenomenon is the anatomy of pelvic blood vessels and the possible presence of anastomoses by which embolic material administered into the uterine arteries may reach the ovarian circulation, and this may decrease the blood supply of the ovary or injure the ovarian tissue directly [25]. Negative impact on ovarian function may be important not only for patients still wishing to conceive, but may also result in premature menopause and significantly deteriorate any patient's quality of life. These concerns have inspired many researchers to investigate the possible influence of UAE on OR. 
The occurrence of menopause after UAE has been reported, but mainly in patients older than 45 years [26]. The FIBROID registry revealed that the incidence of ovarian failure after UAE is approximately $7 \%$, again with the majority of cases occurring in patients over 45 years old [27]. In a study performed on younger patients (all under 40 years old) no significant impact on E2, FSH, and AFC was found at up to five years of follow-up, in comparison to control patients [28]. Spies et al. reported a significant increase in FSH in patients $>45$ years old in comparison to younger patients [29]. However, it has to be stressed that in women younger than 40 years of age, there is evidence that FSH is not a sensitive indicator of changes in $\mathrm{OR}$ and that $\mathrm{AMH}$ is a more sensitive measure that should be used for hormonal assessment in studies of younger patients [30,31]. More recent studies have investigated AMH changes after UAE. McLucas et al. measured $\mathrm{AMH}$ levels before and at least six months after UAE in patients $<40$ years old and did not observe any significant differences [32]. Conversely, Kim et al. reported a significant decrease of $\mathrm{AMH}$ and AFC at three and 12 months after UAE [33]. AMH levels remained low after 12 months of follow-up compared to the expected $\mathrm{AMH}$ levels. However, a significant recovery of $\mathrm{AMH}$ between the three- and 12-month measurements was seen, but only in patients $<40$ years of age [33]. These results seem to confirm the hypothesis that the ovaries may be damaged during UAE, but in younger patients they are likely to recover.

Interestingly, UAE is not the only treatment option for fibroids that may possibly decrease OR. Similar concerns were raised for surgical treatment, mainly hysterectomies [34]. That is why, from a practical point of view, direct comparisons of various treatment techniques for fibroids provide the most important information, allowing selection of the appropriate treatment option for a particular patient. In the randomised EMMY (EMbolisation vs. hysterectoMY) trial the occurrence of elevated FSH levels and menopausal symptoms was similar in both the embolisation and hysterectomy groups at the end of the study (24 months of follow-up) [35]. However, $\mathrm{AMH}$ levels remained significantly decreased only in the UAE group. The authors concluded that the risk of premature ovarian failure is comparable after UAE and hysterectomy, but UAE is more likely to influence the development of follicles and primordial follicle pool [35]. It should be stressed that the mean patient age in this study was approximately 45 years, and this may explain the negative impact of UAE on AMH levels.

Uterine artery embolisation has also been compared with laparoscopic myomectomies [36]. Patients who underwent UAE had significantly lower AMH levels and AFC than patients after myomectomies. FSH and E2 levels were not significantly different between the groups [36]. Mara et al. also assessed the impact of UAE and laparoscopic myomectomy on ovarian function and found a significantly greater number of cases with elevated FSH levels after UAE than after myomectomy [15].

\section{Conclusions and future directions}

Despite the numerous studies already performed, the impact of UAE on fertility is still controversial. Even though pregnancies are possible after UAE and some authors advocate the use of UAE in patients of reproductive age, the risk of obstetrical complications is also clearly reported. That is why current guidelines still consider future reproductive plans as a relative contraindication to UAE. UAE may also lead to ovarian injury and premature menopause, but this phenomenon seems to be most pronounced in older patients.

Nowadays, novel alternative treatment options for fibroids are available. It seems extremely important to directly compare these techniques, to be able to choose the best option for a particular patient. A study comparing UAE with focused ultrasound is currently ongoing [37]. It has recently been shown that the influence of UAE on fibroid volume and vascularity may be comparable to ulipristal acetate - a selective progesterone receptor modulator [38]. There is no data allowing direct comparisons of UAE and ulipristal acetate in terms of fertility issues. However, since ulipristal acetate was shown to be safe and effective in long-term follow-up [39] and many pregnancies were reported after such therapy [40], novel algorithms including ulipristal acetate were proposed for patients $<40$ years of age presenting with symptomatic fibroids and infertility [41]. Future studies should focus on comparing various minimally invasive treatment options for fibroids in terms of the possible impact on fertility and ovarian function.

\section{Disclosure}

Authors report no conflict of interest.

\section{References}

1. Zepiridis LI, Grimbizis GF, Tarlatzis BC. Infertility and uterine fibroids. Best Pract Res Clin Obstet Gynaecol 2016; 34: 66-73.

2. Donnez J, Dolmans MM. Uterine fibroid management: from the present to the future. Hum Reprod Update 2016; 22: 665-686.

3. Blitz MJ, Rochelson B, Augustine $S$, et al. Uterine fibroids at routine second-trimester ultrasound survey and risk of sonographic short cervix. J Matern Fetal Neonatal Med 2016; 29: 3454-3460.

4. Borah BJ, Nicholson WK, Bradley L, Stewart EA. The impact of uterine leiomyomas: a national survey of affected women. Am J Obstet Gynecol 2013; 209: 319.e1-319.e20.

5. Ravina JH, Herbreteau D, Ciraru-Vigneron N, et al. Arterial embolisation to treat uterine myomata. Lancet 1995; 346: 671-672

6. Hickey M, Hammond I. What is the place of uterine artery embolisation in the management of symptomatic uterine fibroids? Aust N Z J Obstet Gynaecol 2008; 48: 360-368. 
7. American College of Obstetricians and Gynecologists. ACOG Practice Bulletin No. 96: Alternatives to Hysterectomy in the Management of Leiomyomas. Obstet Gynecol 2008; 112 (2 Part 1): 387-400.

8. Jacob GP, Oraif A, Power S. When helping hurts: the effect of surgical interventions on ovarian reserve. Hum Fertil 2016; 19: 3-8.

9. Holub Z, Mara M, Kuzel D, et al. Pregnancy outcomes after uterine artery occlusion: prospective multicentric study. Fertil Steril 2008; 90: 1886-1891.

10. Goldberg J, Pereira L, Berghella V, et al. Pregnancy outcomes after treat ment for fibromyomata: Uterine artery embolization versus laparoscopic myomectomy. Am J Obstet Gynecol 2004; 191: 18-21.

11. Homer H, Saridogan E. Uterine artery embolization for fibroids is associ ated with an increased risk of miscarriage. Fertil Steril 2010; 94: 324-330.

12. McLucas B, Voorhees WD 3rd, Elliott S. Fertility after uterine artery em bolization: a review. Minim Invasive Ther Allied Technol 2016; 25: 1-7.

13. McLucas B. Pregnancy following uterine artery embolization: an update. Minim Invasive Ther Allied Technol 2013; 22: 39-44.

14. Mohan PP, Hamblin MH, Vogelzang RL. Uterine artery embolization and its effect on fertility. J Vasc Interv Radiol 2013; 24: 925-930.

15. Mara M, Maskova J, Fucikova Z, et al. Midterm clinical and first reproductive results of a randomized controlled trial comparing uterine fibroid embolization and myomectomy. Cardiovasc Intervent Radio 2008; 31: 73-85.

16. Gupta JK, Sinha A, Lumsden MA, Hickey M. Uterine artery embolization for symptomatic uterine fibroids. Cochrane Database Syst Rev 2014; 12: 4-7.

17. Reed SD, Newton KM, Thompson LB, et al. The incidence of repeat' uterine surgery following myomectomy. J Women's Heal. 2006; 15: 1046-1052.

18. Hamoda H, Pepas L, Tasker F, et al. Intermediate and long-term outcomes following uterine artery fibroid embolization. Eur J Obstet Gynecol Reprod Biol 2015; 191: 33-38.

19. Torre A, Paillusson B, Fain V, et al. Uterine artery embolization for severe symptomatic fibroids: Effects on fertility and symptoms. Hum Reprod 2014; 29: 490-501.

20. Cooper AR, Baker VL, Sterling EW, et al. The time is now for a new approach to primary ovarian insufficiency. Fertil Steril 2011; 95: 1890-1897.

21. Practice Committee of the American Society for Reproductive Medicine Testing and interpreting measures of ovarian reserve: A committee opinion. Fertil Steril 2012; 98: 1407-1415.

22. Cooper G, Baird D, Hulka B, et al. Follicle-stimulating hormone concen trations in relation to active and passive smoking. Obstet Gynecol 1995 85: 407-411.

23. Ryu RK, Chrisman HB, Omary RA, et al. The vascular impact of uterine artery embolization: prospective sonographic assessment of ovarian arterial circulation. J Vasc Interv Radiol 2001; 12: 1071-1074.

24. Aziz A, Petrucco OM, Makinoda S, et al. Transarterial embolization of the uterine arteries: patient reactions and effects on uterine vasculature. Acta Obstet Gynecol Scand 1998; 77: 334-340.

25. Ouyang Z, Liu P, Yu Y, et al. Role of ovarian artery-to-uterine artery anastomoses in uterine artery embolization: Initial anatomic and radiologic studies. Surg Radiol Anat 2012; 34: 737-741.

26. Chrisman HB, Saker MB, Ryu RK, et al. The impact of uterine fibroid embolization on resumption of menses and ovarian function. J Vasc Interv Radiol 2000; 11: 699-703.

27. Spies JB, Myers ER, Worthington-Kirsch R, et al. The FIBROID Registry. Obstet Gynecol 2005; 106: 1309-1318.

28. Tropeano G, Di Stasi C, Amoroso S, et al. Long-term effects of uterine fibroid embolization on ovarian reserve: A prospective cohort study. Ferti Steril 2010; 94: 2296-2300.

29. Spies JB, Roth AR, Gonsalves SM, Murphy-Skrzyniarz KM. Ovarian function after uterine artery embolization for leiomyomata: assessment with use of serum follicle stimulating hormone assay. J Vasc Interv Radiol 2001; 12: 437-442.

30. Munir SS, Sultana M, Jabeen S, Khalid M. Antimullerian hormone as a marker of ovarian reserve. Pakistan J Med Heal Sci 2016; 10: 37-41.

31. Kaump GR, Spies JB. The impact of uterine artery embolization on ovarian function. J Vasc Interv Radiol 2013; 24: 459-467.

32. Mclucas B, Voorhees WD 3rd, Chua KJC. Anti Müllerian hormone levels before and after uterine artery embolization: A preliminary report Minim Invasive Ther Allied Technol 2015; 24: 242-245.

33. Kim C-W, Shim HS, Jang H, Song YG. The Effects of Uterine Artery Embolization on Ovarian Reserve. Eur J Obstet Gynecol Reprod Biol 2016; 206: 172-176.
34. Iwase A, Nakamura T, Nakahara T, et al. Assessment of ovarian reserve using anti-Müllerian hormone levels in benign gynecologic conditions and surgical interventions: a systematic narrative review. Reprod Biol Endocrinol 2014; 12: 125

35. Hehenkamp WJK, Volkers NA, Broekmans FJ, et al. Loss of ovarian reserve after uterine artery embolization: a randomized comparison with hysterectomy. Hum Reprod 2007; 22: 1996-2005.

36. Arthur R, Kachura J, Liu G, et al. Laparoscopic Myomectomy Versus Uterine Artery Embolization: Long-Term Impact on Markers of Ovarian Reserve. J Obstet Gynaecol Canada 2014; 36: 240-247.

37. Bouwsma EVA, Hesley GK, Woodrum DA, et al. Comparing focused ultrasound and uterine artery embolization for uterine fibroids - Rationale and design of the Fibroid Interventions: Reducing Symptoms Today and Tomorrow (FIRSTT) trial. Fertil Steril 2011; 96: 704-710.

38. Czuczwar P, Wozniak S, Szkodziak P, et al. Influence of ulipristal acetate therapy compared with uterine artery embolization on fibroid volume and vascularity indices assessed by three-dimensional ultrasound: Prospective observational study. Ultrasound Obstet Gynecol 2015; 45: 744-750.

39. Woźniak S, Szkodziak P, Czuczwar P, et al. The effect of ulipristal acetate treatment on symptomatic uterine fibroids within 12-months followup. Menopausal Rev 2014; 1: 18-21.

40. Luyckx M, Squifflet JL, Jadoul P, et al. First series of 18 pregnancies after ulipristal acetate treatment for uterine fibroids. Fertil Steril 2014; 102: 1404-1409.

41. Donnez J, Donnez O, Dolmans MM. With the advent of selective progesterone receptor modulators, what is the place of myoma surgery in current practice? Fertil Steril 2014; 102: 640-648. 